\title{
Éditorial:
}

\section{Le point sur la recherche sur l'abus et les mauvais traitements faits aux personnes âgées au Canada}

Ce numéro spécial de La Revue canadienne du vieillissement est consacré à la recherche sur les abus et les mauvais traitements à l'endroit des personnes âgées au Canada. Nous serions heureux d'être les premiers à publier un numéro spécial sur ce sujet au Canada. Mais, des chercheurs et des praticiens canadiens entreprenants, avec la collaboration d'Américains conciliants, ont eu l'occasion d'utiliser un numéro entier du Journal of Elder Abuse and Neglect, en 1992, pour mettre en lumière les plus récentes recherches effectués sur l'abus, les mauvais traitements et la négligence à l'égard des personnes âgées au Canada. Ce numéro rapportait les résultats du fameux sondage national mené par Elizabeth Podnieks qui révélait que 4 pour cent des personnes âgées au Canada, vivant dans un logement privé, avaient été victimes d'une forme quelconque d'abus, de mauvais traitement ou de négligence; de ce pourcentage, 2,5 pour cent déclaraient avoir été victimes d'abus matériel; 1,4 pour cent de violence verbale chronique, 0,5 pour cent de violence physique et 0,4 pour cent de négligence (Podnieks, 1992). La parution de ce numéro spécial de la revue américaine marqua la fin de la première époque de la recherche canadienne sur l'abus, les mauvais traitements et la négligence à l'endroit des personnes âgées. Les chercheurs, incités par des praticiens canadiens, avaient réussi à conscientiser le public canadien à un problème social disgracieux et intolérable.

Les premières études de fréquence menées par Bélanger (1981) et Grandmaison (1988) à Montréal, par Shell (1982) et King (1984) au Manitoba, par Haley (1984) en Nouvelle-Écosse, par Stevenson (1985) en Alberta, et par le ministère des Services sociaux et communautaires (1985) en Ontario, tous des précurseurs du sondage de Podnieks, ont servi à démontrer qu'une certaine proportion des personnes âgées au Canada étaient victimes d'abus. Le premier livre canadien traitant de l'abus et des mauvais traitements faits aux aînés par Schlesinger et Schlesinger (1988) a alerté ceux et celles qui les ignoraient et a suggéré l'application de solutions juridiques mises en oeuvre à Terre-Neuve dès 1973. Au cours des années 1980, des débats théoriques animés ont porté majoritairement sur les lois visant à protéger les adultes ainsi que sur les avantages et désavantages de la dénonciation obligatoire de cas d'abus. Ces discussions ont mené à une réforme du secteur de la protection des adultes. Au cours de cette même décennie, le gouvernement fédéral a participé au financement de diverses initiatives en matière de recherche, d'éducation et d'intervention qui ont permis la réalisation de six grands projets de recherche à la fin des années 80 et au début des années 90 .

Ces efforts des années 80 sont venus à maturité et ont ouvert de nouvelles 
perspectives sur la recherche sur l'abus et les mauvais traitements à l'endroit des personnes âgées au Canada. Contrairement à la période précédente, qui visait à établir des preuves irréfutables de l'existence de l'abus et des mauvais traitements envers les aînés et ce, parfois en exagérant le problème, l'époque actuelle se fonde sur des recherches sérieuses qui fournissent déjà des renseignements pour l'établissement d'interventions pratiques, de politiques et de nouvelles lois éclairées au Canada. Certaines personnes ont avancé que, malheureusement, le Canada tire de l'arrière comparativement aux États-Unis lorsqu'il s'agit de traiter le problème des personnes âgées victimes d'abus, de mauvais traitements ou de négligence. Toutefois, nous pouvons répliquer que le Canada a abordé le problème avec sagesse. En effet, il n'a pas adopté des lois controversées pour la protection des adultes comme il s'en trouve aux États-Unis, mais il a plutôt soigneusement mis à l'essai des approches différentes dans diverses provinces. Les praticiens ont conjugué leurs efforts au sein d'équipes multidisciplinaires en faisant preuve de respect et de sensibilité les uns envers les autres à la lumière des lacunes au plan des connaissances pratiques dans ce domaine. Les gouvernements, quant à eux, ont rapidement promu recherche et formation et, du fait même, encouragé une nouvelle génération de chercheurs. Ce numéro spécial présente un échantillonnage de leurs travaux, qui forment les assises de la recherche à venir et soulèvent certaines des questions importantes que devront régler les Canadiens au cours du $21^{\mathrm{e}}$ siècle qui promet d'être plus frugal, mais, espérons-le, pas plus misérable.

Dans le premier article, Pittaway, Westhues et Peressini étudient, à l'aide de données secondaires, le lien entre les théories habituelles utilisées pour expliquer l'abus, les mauvais traitements et la négligence à l'égard des personnes âgées ainsi que les facteurs de risque d'abus ou de négligence auxquels sont exposés les aînés ou leurs prestateurs de soins. Dans le cadre de leur explication de l'abus à l'égard des personnes âgées, une des lacunes de la recherche est mise en évidence. La recherche d'incidences, soit le nombre de nouveaux cas d'abus pendant une période de temps déterminée, est pratiquement inexistante au Canada et aux États-Unis. Cependant, il s'agit du moyen le plus efficace pour étudier les causes de l'abus et des mauvais traitements chez les ainés, et pour mettre à l'épreuve l'efficacité des programmes de prévention. Les données sur le nombre d'incidents révèlent également si le problème s'aggrave ou non; actuellement au Canada, cette question ne repose que sur des conjectures.

Reconnaissant les limites de leurs données, Pittaway et ses collègues ont constaté que divers facteurs sont en corrélation avec les divers types d'abus. Ils suggèrent qu'il est probablement impossible d'expliquer globalement et entièrement ce phénomène. Cette conclusion est appuyée par de la documentation américaine. En raison de la complexité de la question de l'abus envers les personnes âgées, un certain nombre de théories différentes doivent être utilisées pour en expliquer les diverses dimensions, ce qui n'a pas suscité grand intérêt. Un survol des ouvrages sur l'abus et les mauvais traitements à l'endroit des personnes âgées permet de constater qu'il n'existe 
aucune nouvelle théorie. Bien que la théorie féministe, sous ses nombreuses formes, constitue un ajout important dans le domaine, il ne s'agit pas réellement d'une nouvelle théorie, mais plutôt d'un point de vue récent emprunté du secteur de la violence dans la famille. Fait assez étrange, la forme d'abus la plus courante au Canada, soit sur le plan financier, est moins étudiée dans les milieux universitaires sur le plan théorique que les autres formes d'abus. Aujourd'hui, de plus en plus de résultats de recherches mettent l'accent sur les aspects interactifs des comportements abusifs envers les aînés et appuient les distinctions faites entre les mauvais traitements faits aux personnes âgées, les mauvais traitements faits par les aînés et les mauvais traitements mutuels. Toutefois, peu d'efforts ont été consacrés à l'élaboration d'une théorie à la lumière de ces récentes découvertes. S'abstenir de formuler de nouvelles théories se fait à nos propres risques, même si cette aventure soulève le scepticisme, car la théorie détermine les initiatives à entreprendre pour régler le problème d'abus et de mauvais traitements et se reflète dans les politiques sociales et les lois.

Bien que les progrès sur le plan théorique se soient réalisés lentement, l'élaboration d'un protocole de dépistage est une voie très prometteuse, comme le démontre l'article de Reis et Nahmiash. Dans le cadre de leur étude, ils ont mis à l'épreuve la validité du test de dépistage de comportements abusifs chez les prestateurs de soins (Caregiver Abuse Screen CASE), qui consiste en un questionnaire de huit questions auxquelles doivent répondre les prestateurs de soins. Cette étude, adroitement conçue, démontre que la méthode de dépistage peut faire la distinction entre les personnes victimes d'abus et celles qui ne le sont pas, et constituera un outil très apprécié dans la panoplie de moyens dont disposent les praticiens pour lutter contre l'abus fait aux personnes âgées. Ceci dit, il reste encore beaucoup de travail psychométrique à réaliser au Canada en vue d'élaborer des méthodes de dépistage de comportements abusifs ou de mauvais traitements à l'égard des personnes âgées ainsi que des personnes susceptibles d'être victimes d'abus. Il est également nécessaire d'établir un protocole de classification pour aider à faire un diagnostic et intervenir adéquatement.

Dans un même ordre d'idées, l'article de Stones et Pittman présente un autre exemple des activités de recherche consacrées à l'élaboration de caractéristiques psychométriques pour mesurer les attitudes vis-à-vis de l'abus et des mauvais traitements faits aux personnes âgées. À l'aide de deux études, Stones et Pittman confirment que le test d'attitudes vis-à-vis de l'abus et des mauvais traitements faits aux personnes âgées (EAAT) représente une mesure adéquate sur le plan psychométrique pour déterminer les différences d'attitudes vis-à-vis de l'abus et des mauvais traitements faits aux aînés. De plus, les auteurs discutent de résultats de recherche fascinants qui soulèveront beaucoup de controverses dans certains quartiers. Leurs recherches ont révélé que les personnes susceptibles d'adopter des attitudes extrêmes sont davantage persuadées que la société a la responsabilité de mettre fin aux abus. Les auteurs soulignent qu'une attitude extrême est liée à des convictions simplistes et conflictuelles, et que ces tendances 
présagent des attitudes encore plus extrêmes vis-à-vis des personnes extérieur au groupe. Par exemple, les jeunes praticiens ont évalué des exemples d'abus et de mauvais traitements faits aux aînés plus sévèrement que les personnes âgées. Ces résultats, notamment, peuvent servir à expliquer la raison pour laquelle certains aînés victimes d'abus choisissent un mode de vie plus risqué que celui que d'autres personnes choisiraient pour eux. Les auteurs soulèvent la question à savoir si les défenseurs s'opposant le plus à l'abus possèdent des convictions simplistes et conflictuelles. Mis en pratique, leurs résultats peuvent aider à expliquer les critiques dirigées contre les recherches passées sur l'abus, qui étaient perçues comme des exagérations du problème et des appels à des mesures draconiennes.

L'article d'Aronson, Thornewell et Williams est un exemple de l'importance de classer les types d'abus correctement. Ces auteurs présentent le témoignage d'une femme ayant quitté son mari violent lorsqu'elle était dans la soixante-dizaine. Ils soutiennent que l'abus et les mauvais traitements faits aux personnes âgées doivent être présentés sous leur vrai jour dans nombre de cas, soit une agression contre l'épouse. Selon eux, il s'agit d'un problème voilé en raison de la neutralité des sexes en gérontologie et du refus d'accorder de l'importance à l'âge dans les études sur les femmes. Même si les études d'incidences actuelles démontrent clairement que la violence faite aux épouses représente une composante importante de l'abus et des mauvais traitements à l'égard des personnes âgées, les gérontologues sont encore peu disposés à délaisser les modèles de réaction qui considèrent le mauvais traitement comme une réponse irrationnelle à une situation de prestation de soins stressante. Ce modèle est enraciné dans la littérature sur la violence familiale, envers les enfants en particulier, et peut être considéré comme s'opposant aux interprétations féministes de la violence, qui sont définitivement mieux équipées pour définir la violence faite aux épouses. En se fondant donc sur le modèle de réaction, on comprend pourquoi les gérontologues soient peu enclins à identifier les conjoints comme étant responsable de comportements abusifs parce que les prestateurs de soins auprès de la famille, c'est-à-dire les femmes, représentent le pilier des soins communautaires auprès des personnes âgées. Les recherches canadiennes révèlent indubitablement que les membres de la famille sont des prestateurs de soins de bonne volonté et responsables; il est donc difficile de les accuser d'abus. De plus, l'abus du conjoint requiert parfois des solutions différentes qui ne font pas partie du lexique d'intervention des gérontologues (McDonald, Hornick, Robertson, \& Wallace, 1991).

Quelles que soient les raisons qui font hésiter les gérontologues à reconnaitre les mauvais traitements, traditionnels ou nouveaux, aux épouses d'âge avancé, l'application des théories féministes à toutes les formes de mauvais traitements entre conjoints demeure toujours une question controversée. Par exemple, les résultats de recherche de plus en plus nombreux sur la violence conjugale dans les couples homosexuels a remis en question les théories de violence entre partenaires fondées sur le sexe (Renzetti, 1994). Selon ce point de vue, le vrai coupable est l'iniquité des pouvoirs en- 
tre les partenaires. Les théories féministes pourraient alors être élargies pour expliquer l'abus et les mauvais traitements faits aux conjoints et aux conjointes si elles étaient conçues en ces termes car actuellement, le nombre de déclarations de mauvais traitements à l'égard de personnes âgées est incomplet parce que les hommes plus âgés sont tout autant susceptibles d'être victimes de mauvais traitements que les femmes. Aronson et ses collègues avancent l'hypothèse que des théories avancées pourraient également être directement appliquées dans le cas d'autres groupes plus âgés et vulnérables, par exemple les personnes appartenant à une autre ethnie et les personnes âgées très frêles. Plus important encore, les théories féministes mettent en lumière les conséquences plus globales au point de vue du sexe, de la race, de l'ethnie et de la classe sociale, qui sont tous des facteurs structurels trop souvent laissés de côté dans le cadre de l'étude de mauvais traitements faits aux personnes âgées.

Les trois derniers articles de ce numéro spécial abordent les préoccupations constantes des Canadiens en ce qui touche les lois pour la protection des adultes. L'article de Gordon décrit l'évolution des lois canadiennes sur l'abus et les mauvais traitements faits aux personnes âgées en décrivant les trois vagues de réformes successives des lois régissant les tutelles. L'auteur se préoccupe particulièrement de la troisième vague de réformes, qui consiste en l'adoption récente des nouveaux statuts omnibus de tutelle des adultes, comprenant les dispositions de protection des adultes. Il soulève trois problèmes possibles que devront régler les Canadiens, surtout à la lumière des articles de Poirier et de Bond, Penner et Yellen qui appuient empiriquement les observations de Gordon. La première question porte sur l'amalgame des tutelles découlant d'une ordonnance du tribunal et les dispositions de protection sous les mêmes statuts. Cette combinaison peut comporter des éléments positifs mais, d'un autre côté, les tutelles par ordonnance, une solution intrusive à l'abus et aux mauvais traitements faits aux personnes âgées, risquent de devenir une décision de routine. Une des suggestions proposées par Gordon afin de prévenir ce problème consiste à réserver le rôle des tribunaux. L'article de Poirier, qui compare les résultats pour les clients assujettis aux lois de protection des adultes du NouveauBrunswick, révèle que la décision d'ordonner des mesures de protection dépend de la représentation du client par un avocat. Les données, fondées sur des analyses de cas de justice entre 1983 et 1989, démontrent clairement que les clients âgés obtiennent de meilleurs résultats s'ils sont représentés par un avocat. Dans un même temps, Poirier souligne que la ligne de pensée du juge par rapport à la loi a une incidence importante sur le résultat de la cause. Toutefois, il y a un risque que certains juges acceptent plus facilement l'orientation vers l'intervention sociale qui domine, malheureusement, la loi sur la protection des adultes.

Gordon souligne que les plus récentes lois touchant le rôle important de la représentation pour protéger les adultes victimes d'abus ou de mauvais traitements en constituent le deuxième point faible. À l'heure des compressions fiscales, il est fort possible que ces services raffinés, offerts à une po- 
pulation particulière, seront réduits par les politiciens, qui effectuent d'importantes compressions budgétaires au nom du déficit. Dans le cadre d'un sondage auprès des professionnels sur les lois concernant l'abus et les mauvais traitements faits aux personnes âgées, Bond, Pepper et Yellen ont constaté que les répondants se préoccupaient majoritairement de l'insuffisance des fonds pour administrer ces lois et offrir des services aux personnes âgées victimes d'abus ou de mauvais traitements bien avant que le gouvernement décide de réduire sérieusement le fardeau de la dette! La dernière question soulevée par Gordon, soit la possibilité d'un accroissement de cas d'abus et de mauvais traitements faits aux aînés en raison de l'adoption de lois retirant certaines mesures de protection qui favorisent l'habilitation (empowerment) des aînés ne repose sur aucune référence empirique. Les lacunes en recherche sur les avantages et désavantages de l'habilitation (emprunté du mouvement pour les handicapés intellectuels) et de la protection des personnes âgées rappellent un peu la mise en application hâtive des lois de déclarations obligatoires (empruntées des initiatives préconisant le bienêtre des enfants) avant d'en connaître la portée. L'habilitation des personnes âgées est devenue une approche influente en gérontologie, mais nous devons nous assurer qu'elle ne devienne pas un euphémisme pour aucun service ou aucune protection.

En faisant le point sur la recherche canadienne en matière d'abus, de mauvais traitements et de négligence à l'égard des personnes âgées, il est encourageant de constater les importants progrès réalisés en si peu de temps par un petit groupe de chercheurs. Il ne faut cependant pas croire que la recherche sur l'abus, les mauvais traitements et la négligence reçoit l'attention qu'elle mérite ou le degré de sérieux qu'elle requiert. Les chercheurs canadiens doivent réaliser un programme de recherche de taille en disposant de moins en moins de ressources financières. Malheureusement, le coût des recherches à effectuer seront élevés. À tout le moins, nous devons réaliser les projets suivants: une étude d'incidences des abus et des mauvais traitements faits aux personnes âgées; une étude des fréquences d'abus et de mauvais traitements faits aux personnes âgées dans les établissements afin de déterminer qui abuse qui; une étude des fréquences d'abus et de mauvais traitements aux personnes âgées dans le cadre des services de soins à domicile; des études de cas-témoin afin de déterminer les facteurs de risque à l'abus; plus de recherches d'évaluation de nature qualitative et quantitative pour déterminer les mesures qui fonctionnent en pratique; l'élaboration et la mise à l'essai continues de test de dépistage et de catégories de classement; de même que des évaluations directes des derniers changements apportés aux lois. Ces initiatives devront être entreprises dans un climat où l'utilisation et la pertinence de la recherche sont souvent remises en question car on considère que les fonds pourraient servir à de meilleures fins en matière de prévention et de prestation de services. Qui plus est, l'abus et les mauvais traitements à l'égard des personnes âgées n'est que l'un des nombreux problèmes sociaux tandis que la concurrence pour des fonds de recherche de plus en plus rares se fait plus féroce dans un contexte où les 
agences de financement spécialisées sont dissoutes.

Les obstacles sont-ils insurmontables? Nous ne le croyons pas. La recherche sera nécessaire en vue d'aider à rationner l'utilisation des maigres ressources destinées aux interventions, aux services et aux politiques sociales en démontrant leur efficacité. La majorité des Canadiens se préoccupent du destin des aînés au Canada. Les gouvernements, même s'ils éprouvent des contraintes financières, continueront probablement de soutenir la recherche pratique. La recherche sur l'abus et les mauvais traitements s'insérera probablement dans la perspective du cycle de vie, et les chercheurs canadiens continueront de faire preuve de créativité et d'ingéniosité.

\section{Références}

Bélanger, L. (1981). The types of violence the elderly are victims of: Results of a survey done with personnel working with the elderly. Document présenté lors de la $34^{\mathrm{e}}$ Réunion scientifique annuelle de la Société de gérontologie de l'Amérique, à Toronto, au Canada.

Grandmaison, A. (1988). Protection des personnes âgées: Étude exploratoire de la violence à l'égard de la clientèle des personnes âgées. Manuscrit non publié. Montréal, Centre de Services Sociaux du Montréal Métropolitain (CSSMM).

Haley, R.C. (1984). Elder abuse/neglect. Halifax: Département des Services sociaux.

King, N.R. (1984). Exploitation and abuse of older family members: An overview of the problem. Dans J.J. Costa (Éd.), Abuse of the elderly: A guide to resources and services (pp. 56-68). Lexington, MA: Lexington Books.

McDonald, L., Hornick, J., Robertson, G., \& Wallace, J. (1991). Elder abuse and neglect in Canada. Toronto: Butterworths.

Ministère des Services sociaux et communautaires. (1985). Report of a survey of elder abuse in the community. Toronto: Comité permanent sur le développement social, Gouvernement de l'Ontario.

Podnieks, E. (1992). National survey on Abuse of the elderly in Canada. Journal of Elder Abuse and Neglect, 4, 5-58.

Renzetti, C.M. (1994). On dancing with a bear: Reflections on some of the current debates among domestic violence theorists. Violence and Victims, 9, 195-200.

Schlesinger, B., \& Schlesinger, R. (1988). Abuse of the Elderly: Issues and Annotated Bibliography. Toronto: University of Toronto Press.

Shell, D.J. (1982). Protection of the elderly: A study of elder abuse. Rapport du Conseil manitobain sur le vieillissement. Winnipeg: Association de gérontologie.

Stevenson, C. (1985). Family Abuse of the Elderly in Alberta. Un rapport du ministères des Services sociaux et de Santé communautaire de l'Alberta, Bureau des aînés.

Lynn McDonald et Blossom Wigdor 